\title{
Evidence for Efficacy of Combination of Antiepileptic Drugs in Treatment of Epilepsy
}

\author{
Ehsan M. Sarhan ${ }^{\mathrm{a}, \mathrm{b}}$, Matthew C. Walker ${ }^{\mathrm{a}}$, Caroline Selai ${ }^{\mathrm{a}}$
}

\begin{abstract}
Epilepsy is the tendency to have recurrent, unprovoked seizures. Although $70 \%$ of epileptic seizures can be controlled with monotherapy (treatment by single antiepileptic drug), a combination of two or more anti-epileptic drugs (AEDs) may be required to improve efficacy (seizure control) and tolerability. Polytherapy (treatment with two or more AEDs) can affect efficacies and side effects in additive, supra-additive (synergistic) or infra-additive fashion. The effect is considered supraadditive when the efficacy of the combination is greater than the sum of the individual drug efficacies, while it is considered infra-additive when the efficacy of the combination is less than the sum of the individual drug efficacies. Here, we have reviewed the available studies and evidences for the application of polytherapy in humans and animal models, to understand which combination of AEDs act as a synergistic polytherapy for epilepsy. We have searched the bibliographic databases MEDLINE and PubMed for studies conducted from 1950 to 2013 and have concluded that, although promising results from the experimental point of view support the combinations of topiramate separately with lamotrigine, gabapentin and felbamate, the most reliable evidence supports the use of valproate and lamotrigine, as this combination generates encouraging results in animal models. Though effectiveness of this combination is supported by human data, there is the possibility of increased side effects. The new drugs are all effective as add-on therapy; there is some evidence that at present, in clinical practice, levetiracetam and topiramate may be the most effective add-on therapies in partial and some generalized epilepsies.
\end{abstract}

Keywords: Antiepileptic drugs; Polytherapy; Combination therapy; Isobolography; Add-on therapy

\section{Introduction}

The hypothesis that a combination of drugs offers advantages

Manuscript accepted for publication November 10, 2015

aUCL Institute of Neurology, Queen Square, London WC1N 3BG, UK ${ }^{b}$ Corresponding Author: Ehsan M. Sarhan, UCL Institute of Neurology, Queen Square, London WC1N 3BG, UK. Email: e.sarhan.12@ucl.ac.uk

doi: http://dx.doi.org/10.14740/jnr356w over monotherapy has been illustrated in a variety of medical fields, including epilepsy. In 1912, Hauptmann introduced phenobarbital (PB), which was used in combination with bromide. In 1953, Yaher and his colleagues reported that phenytoin (PHT) was more successful than PB, and also that the combination of two drugs was more effective in controlling seizures than either used alone [1]. In 1956, phelantin, a tablet composed of PHT, PB and methamphetamine, became available [2]. In the early 1980s, monotherapy was considered to be better than polytherapy in terms of patient compliance to take the medication regularly and associated adverse effects $[3,4]$. Partial seizures in $35-60 \%$ of adults can be controlled with monotherapy. This rate is higher by $10-20 \%$ for adults with tonic-clonic (grand-mal) seizure [5-7]. AEDs with delayed onset of action are less effective and favorable for patients because seizure reduction and/or cessation take longer, although patients have responded from such treatment $[5,8]$. Although the initial monotherapy successfully treats approximately half the patients with epilepsy, subsequent trials of monotherapy are less successful; overall, only $60-70 \%$ of patients become seizure free through monotherapy. When the initial drug fails to control the epilepsy, adding-on alternative AEDs and gradually titrating up becomes necessary. The aim is to taper the first anti-epileptic drug to obtain monotherapy again. If the additional drug fails to manage the seizures, other alternative AEDs are recommended until seizures are controlled $[9,10]$. Although most AEDs are hindered by side effects, the idea of combining two or more AEDs is to improve the efficacy (seizure control) and tolerability of the treatment and to obtain better control of the refractory seizure [11]. A combination of AEDs can produce different efficacies and side effects in either an additive, supra-additive (synergistic) or infra-additive fashion $[7,12]$. It is considered supra-additive when the efficacy of the combination is greater than the sum of the individual drug efficacies, while it is considered infra-additive when the efficacy of the combination is less than the sum of the individual drug efficacies [13]. Polytherapy is more expensive than monotherapy. Furthermore, patients may find it more difficult to manage and comply with polytherapy than with monotherapy, which in turn may lead to failure to control seizures. Although polytherapy is prescribed when monotherapy fails to control seizures, unfortunately there have been insufficient clinical trials to determine the best combinations of AEDs. Therefore, the rational for combinations of AEDs is based on theoretical 


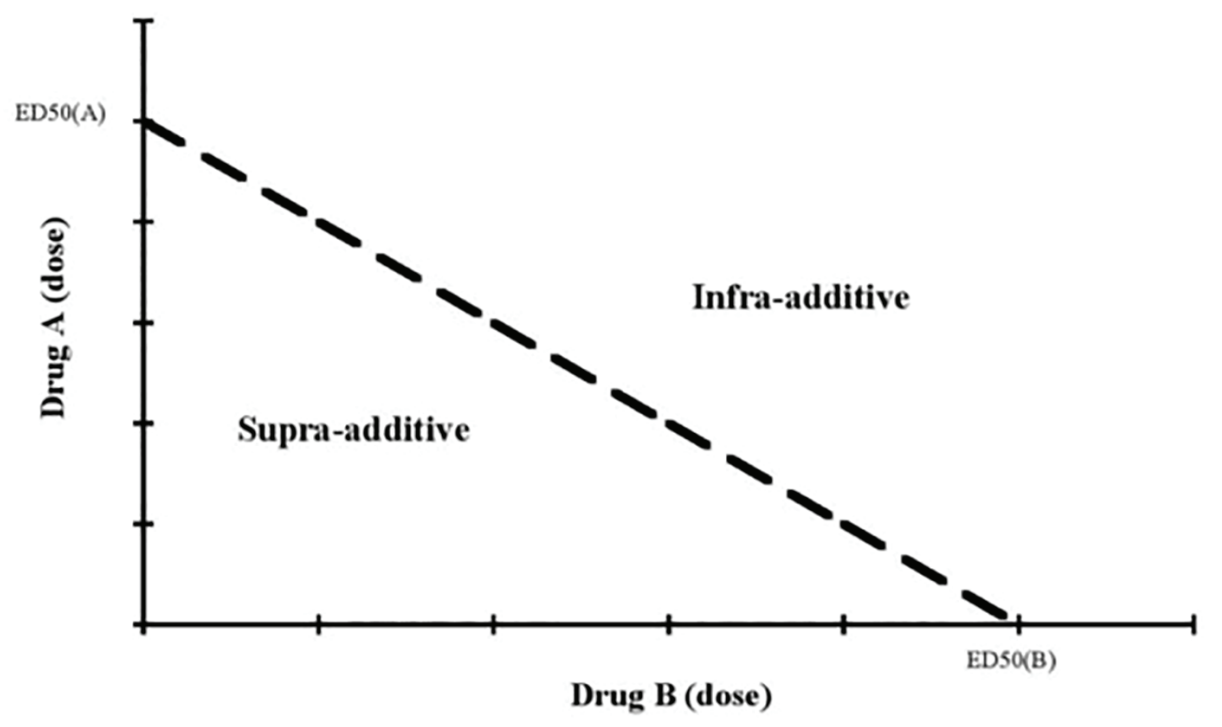

Figure 1. The isobolographic analysis of pharmacodynamic interactions [13].

considerations and animal data.

\section{Animal Evidence for Synergistic Combinations}

The history of using animal models in experimental investigation of efficacy and safety against seizures began in 1937 1938 and resulted in the introduction of PHT into clinical use. In 1955, early experimental studies exploring combinations of established AEDs were reported, such as PB and PHT, which were administrated to rats individually and in various combinations and were tested in the maximal electroshock seizure (MES) model [14]. The pharmacodynamic interactions are more difficult to recognize and measure than pharmacokinetic interactions and can be identified only when pharmacokinetic interactions have been ruled out [15]. Experimental studies have attempted to evaluate the nature of these interactions by applying basic convulsive tests, such as the pentylenetetrazole test (a model of myoclonic seizures), the maximal electroshock test in rats (a model of tonic-clonic seizures in humans to a certain degree), and amygdala-kindled convulsions (a model of partial seizures) [16]. Generally, the available experimental data have come from the maximal electroshock test through isobolographic analysis; some results have also been provided by the pentylenetetrazole test and isolated data have been recovered from amygdala-kindled seizures in rodents [17]. The experiments study combinations of conventional AEDs by themselves as well as combinations of conventional AEDs with novel AEDs. The assessment of drug combinations in the animal models has pitfalls and is not easy. According to Walker, "simply adding a sub-therapeutic dose of one drug to another and showing efficacy tells us nothing about the potency of a combination. Combining two sub-therapeutic doses of the same drug can result in a therapeutic effect" [13]. This has opened the door to a more sophisticated method of assessing drugs combinations, known as isobolographic analysis.
Isobolographic analysis has been in use since the middle of the last century [18]. It provides a substantial basis for assessing whether biological interactions and responses induced by combining two or more agents are more than, equal to, or less than what would have been expected on the basis of individual activities of the component agents and the dose additivity [19].

Currently, it is widely used to determine the pharmacodynamic, biochemical, and physiological effects of drugs on the body in empirical animal studies, particularly for AEDs. It can be represented as a graph in which concentrations of two drugs (A and B) are contrasted and plotted. The effective doses of the administered drugs are individually plotted on the graph. A specific median-effective concentration or dose that results in protection against induced seizures in $50 \%$ of animals for the individual drugs and combinations (ED50, EC50) is plotted on the graph. If the combination is equally additive to the sum of the individual drug efficacies, the points representing the dose of combined drugs will fall in a straight line connecting $\mathrm{A}$ and $\mathrm{B}$. If a combination of drugs is supra-additive, i.e. the efficacy of the combination is greater than the sum of the individual drug efficacies, its point will be below the straight line. Conversely, if a combination is infra-additive, i.e. the efficacy of the combination is less than the sum of the individual drug efficacies, its point will lie above this line [13, 20,21] (Fig. 1). Another approach to assess the pharmacodynamic interactions of AEDs is to add sub-protective dose of one AED (A) to the ED50 value of another AED (B) against induced (empirical) seizure. This ED50 of the second AED is compared to the control ED50 value [22].

\section{Animal data}

In empirical studies on animals, all existing AEDs (conventional and novel) are effective in at least one of the convulsive tests, such as the maximal electroshock and the pentylenetetra- 
zol-induced acute seizures, with the exception of levetiracetam [22-24]. Although assessing a pharmacokinetic interaction is relatively straightforward, evaluation of pharmacodynamics interactions between two drugs requires a valid quantitative assessment [20]. Results regarding animal evidence of combinations have been described in 27 papers, which have been summarized in Table 1.

\section{Human evidence for synergistic combinations}

Polytherapy (combination therapy) has a poor reputation compared to monotherapy (single therapy), for good reasons. Combination therapy can result in poor compliance, drug interactions, and increased side effects [13]. The old arguments against polytherapy were built upon the observation that patients with refractory seizures, when placed in combination therapy, experienced increased side effects without improved efficacy [25]. However, polypharmacy has experienced renewed interest since the 1980s [26]. A deeper understanding of the mechanisms of AEDs and the monitoring of levels of AEDs has led to more effective use of rational polytherapy. Such combinations of drugs can be used to combine mechanisms of action, so that the efficacy of the combinations of AEDs is supra-additive or additive. Furthermore, monitoring of AED levels can limit pharmacokinetic variations which cause adverse effects when AEDs are used in combination [27]. Unfortunately, there have been very few trials that study the benefit of AED combinations. There is no direct method for investigating pharmacodynamic interactions of AEDs in the clinical arena [28]. The isobologram analysis method has not been used in studies on humans because it is impossible to titrate identically in all treatment groups [29]. The common studies on the additive effects of AEDs are the randomized placebo-controlled add-on studies of the novel AEDs. The patients participate in these studies (trials) on a variety of baseline AEDs, usually with a maximum of two. Due to the small number of patients on each baseline AED, the studies have not been sufficiently powered to gather specific information about the effectiveness of drug combinations [27]. Although efficacy in such trials can be observed as a measure of the value of polytherapy, it is not clear whether additional efficacy is due to the effect of added-on AED alone or its combination with other AEDs [13].

\section{Human data}

Unfortunately, available clinical studies on the combinations of AEDs are significantly limited. One significant problem with clinical studies on combinations is that drug load is not taken into consideration. Adverse effects of certain drugs combined at lower doses would not exist when the same drugs are combined at higher doses [27]. Deckers et al have pointed out that a clinician should take drug load into account, and has further used a prescribed daily dose and defined daily dose (PDD/ DDD) calculation to measure drug load. In this review, they have also observed that most clinical trials of add-on therapy do not give information about doses of background drugs, so it is difficult to estimate the total drug load [30]. Efficacy in these trials cannot be considered as evidence of value of polytherapy; however, it is not clear whether the additional effect is due to the added individual agent or its combination with other AEDs [13].

\section{Discussion}

Whenever patients have taken two or more drugs simultaneously, there is the potential of some kinds of pharmacodynamic interaction. It is assumed that these interactions (efficacies) can be additive, supra-additive or infra-additive. If the combined efficacy $\mathrm{C}$ of drugs $\mathrm{A}$ and $\mathrm{B}$ administrated together is equal to the expected efficacies of drug $\mathrm{A}$ and drug B individually, the interaction is considered to be additive. If the combined efficacy is greater than the expected efficacies of drug A and drug B individually, the interaction is assumed to be supra-additive (potentiation). The term synergism can be used synonymously with supra-additive efficacy. In cases when the combined efficacy is less than the expected efficacies of drugs A and B individually, the interaction is presumed to be infra-additive (antagonistic) [31]. Two important questions are raised in the light of the above: What is the combined efficacy of two or more drugs and how is it evaluated? The experimental and clinical studies address these questions regarding the therapeutic effects of various drug combinations.

\section{Experimental studies}

\section{Interactions among classical AEDs}

There have been many experimental studies on animals regarding combinations of conventional AEDs. For example, Bourgeois, Masuda, Chez, and Weaver have assessed the interactions between conventional AEDs in animal models with the pentylenetetrazol and maximal electroshock tests [22]. A combination of PHT and PB has been found to have a clearly additive anticonvulsant effect against maximal electroshock in mice, while the neurotoxic effect has been observed to be infra-additive, based on brain concentration of both AEDs in rats. However, the therapeutic index of PHT alone has been found to be higher than that of the combined treatment [32]. Other data have also pointed to a synergy for this particular combination, but the calculation of therapeutic index seems to be impossible because the neurotoxicity has not been evaluated [33]. For the same AED combination, Weaver et al have reported purely synergism in terms of anticonvulsant effect and infra-additivity with regard to neurotoxicity [34]. In the empirical studies on animals, valproate seems to be more promising when co-administered with conventional AEDs.

If we look at the studies which had been reported by Bourgeois and Chez, we can see additive, supra-additive and additive anticonvulsant effects when valproate was combined with $\mathrm{PB}, \mathrm{PHT}$ and carbamazepine $(\mathrm{CBZ})$ respectively in maximal electroshock test in mice. Regarding the neurotoxic effects of these combinations, additive, infra-additive and additive 
Table 1. The Evidences of Combination of AEDs in Animal Models

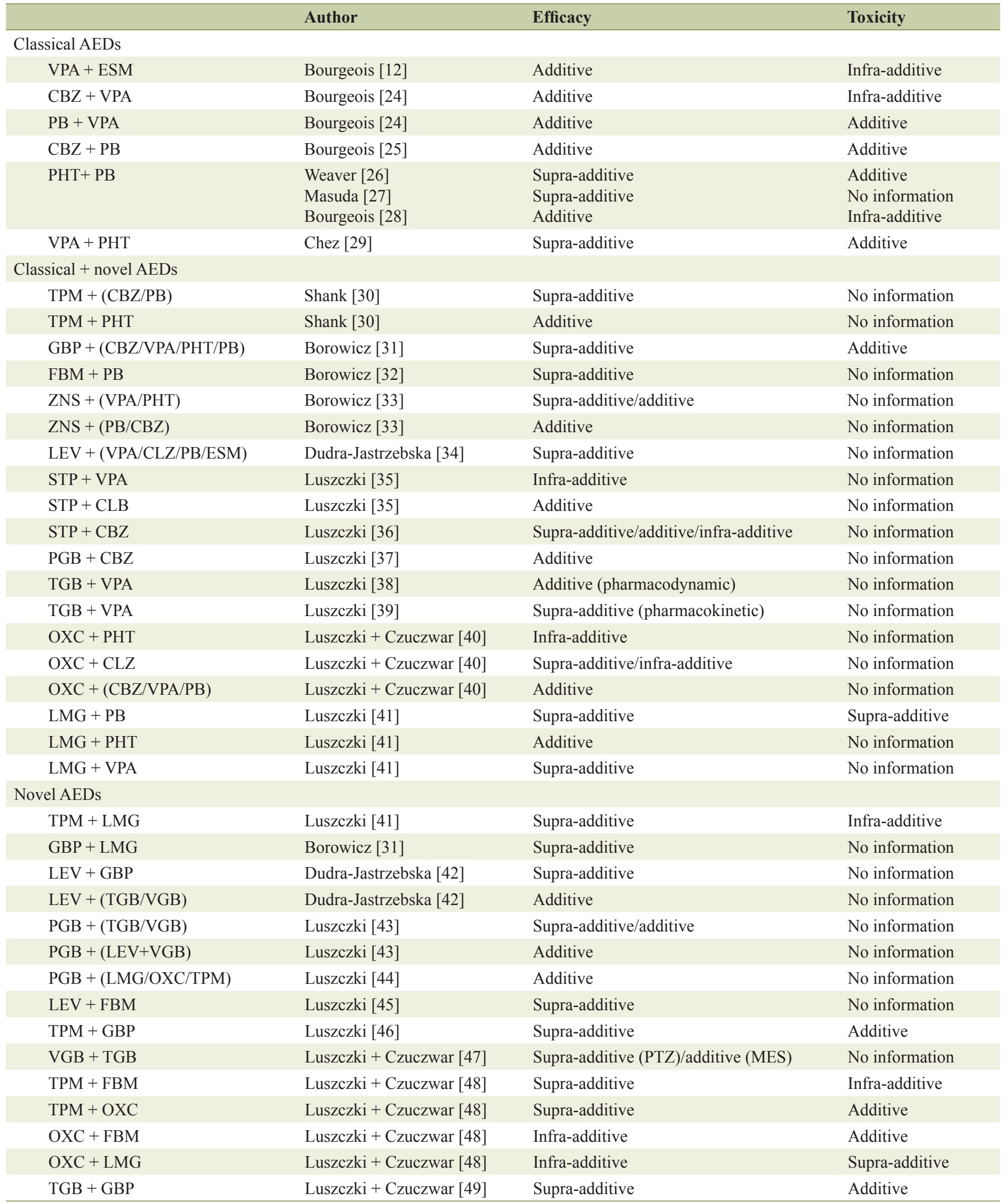


effects were evident respectively $[35,36]$. In the pentylenetetrazole test, valproate has been co-administrated with ethosuximide and observed to exert an additive effect. The neurotoxicity is simply infra-additive, which might indicate that increased anticonvulsant efficacy (effectiveness) is often due to enhanced tolerability [12]. A less promising combination has been found between $\mathrm{CBZ}$ and PB which has an additive effect in both efficacy and toxicity [37].

\section{Interactions between classical and novel AEDs}

One of the initial studies considering the evaluation of interactions between classical and novel AEDs by isobolographic analysis through maximal electroshock test in mice was published by Shank in 1994. The combinations of topiramate with PB or CBZ had a synergistic anticonvulsant effect, while those with PHT were additive. Because neurotoxicity of these combinations was not assessed and no pharmacokinetic studies (estimating the concentrations of AEDs in the brain or plasma) were conducted, it is difficult to evaluate the significance of these results [38]. Notably, combinations of gabapentin with many novel AEDs (valproate, CBZ, PB and PHT) produced obvious anticonvulsant synergy against maximal electroshock in mice. The neurotoxicity effects were only additive. Consequently, another question arises: Does the same AED combination produce different pharmacodynamic interactions at different doses? Studies have reported that completely different interactions, including synergistic and antagonistic anticonvulsant effects, can occur with a particular AED combination, depending on the drug ratio. This may be explained by multiple mechanisms of action of individual AEDs. For example, clonazepam, when combined with oxcarbazepine in the maximal electroshock test at dose ratios of $1: 2$ and $1: 1$, produced anticonvulsant synergy; when the dose ratio was changed to $3: 1$ or $4: 1$, however, anticonvulsant antagonism was exerted. When oxcarbazepine was co-medicated with PHT, at the dose ratio of 1:1, an infra-additive anticonvulsant effect was found; other dose ratios yielded an additive effect [39]. Also, a synergistic interaction was reported for the combinations of zonisamide with valproate and zonisamide with PHT at a fixed dose ratio of $1: 1$, while an additive effect was found for their combinations at fixed dose ratios of $1: 3$ and $3: 1$. In contrast, interactions between zonisamide and $\mathrm{CBZ}$ or $\mathrm{PB}$ at all dose ratios proved to be additive. No effect regarding neurotoxicity was reported. Brain zonisamide concentration was reduced by PB and increased by PHT [40]. Although the combination of lamotrigine and $\mathrm{PB}$ proved to be synergistic against maximal electroshock in mice, a synergy was also found in terms of neurotoxicity, making this combined treatment unfavorable. However, promising results have been found for combined administration of valproate with lamotrigine against maximal electroshock test in mice, while the interaction was antagonistic in the chimney test (a measure of motor performance). The combination was synergistic in the dose ratio of 1:1 [41]. Levetiracetam was combined synergistically with classical AEDs (valproate, ethosuximide clonazepam, and PB) in the pentylenetetrazole test in mice, with no reported adverse effects. Only ethosuximide significantly reduced the brain concentra- tion of levetiracetam; no other pharmacokinetic changes were found [42]. Some combined treatments may be confounded by pharmacokinetic treatments, which can influence the efficacy of AED combinations. For instance, in the maximal electroshock test in mice, stiripentol was combined with CBZ to produce three different interactions dependent on dose of ratios. A synergistic interaction was found at dose ratio of $3: 1$, additivity was observed at a ratio of $1: 1$, and infra-additive was found at a ratio of $3: 1$. The pharmacokinetic interaction of this combination elevated the concentration of CBZ significantly in the brain. No adverse effects were reported for this combination [43]. Another pharmacokinetic interaction was associated with the combination of tiagabine and valproate, which was synergistic in the maximal electroshock test [44]. The same combination in the pentylenetetrazole test proved to be additive. This combination tended to be positive from preclinical point of view [45].

\section{Interactions among novel AEDs}

The pharmacodynamic interactions between novel AEDs have promising results and were exemplified in combination outcomes of novel AEDs. For example, the combination of topiramate and lamotrigine at the dose of 1:1 is favorable, as anticonvulsant supra-additivity and neurotoxic infra-additivity are observed [41]. A combination of topiramate and gabapentin can be considered as a positive one as its anticonvulsant effect is synergistic and its neurotoxicity is additive [46]. Encouraging results are observed with combinations of topiramate with felbamate at three dose ratios; this interaction has a synergistic anticonvulsant action and an antagonistic neurotoxic effect [47]. Conversely, the worst combination between novel AEDs in terms of neurotoxic and anticonvulsant effects is that of oxcarbazepine and lamotrigine in the maximal electroshock test in mice at the dose ratio of $1: 1$, in which synergistic neurotoxic and antagonistic anticonvulsant effects are reported [47]. Generally, the combinations between novel AEDs are free from pharmacokinetic interactions. However, an exception to this rule is the combination of gabapentin and lamotrigine. Although this combination has a positive preclinical profile (anticonvulsant synergy associated with lack of adverse effects), lamotrigine increases the brain concentration of gabapentin [48].

\section{Clinical studies}

\section{Combined AEDs}

Whenever an AED combination is proven to have enhanced effectiveness in animal models, such a combination should be practically tested in human [29]. Deckers et al have indicated that the dosage of an individual drug should be increased to the maximum tolerated dose and then other AEDs should be added [30]. Two study designs are applicable for clinical studies. In the first design, patients who do not respond to drugs A or B individually are administered a combination of A and B. The 


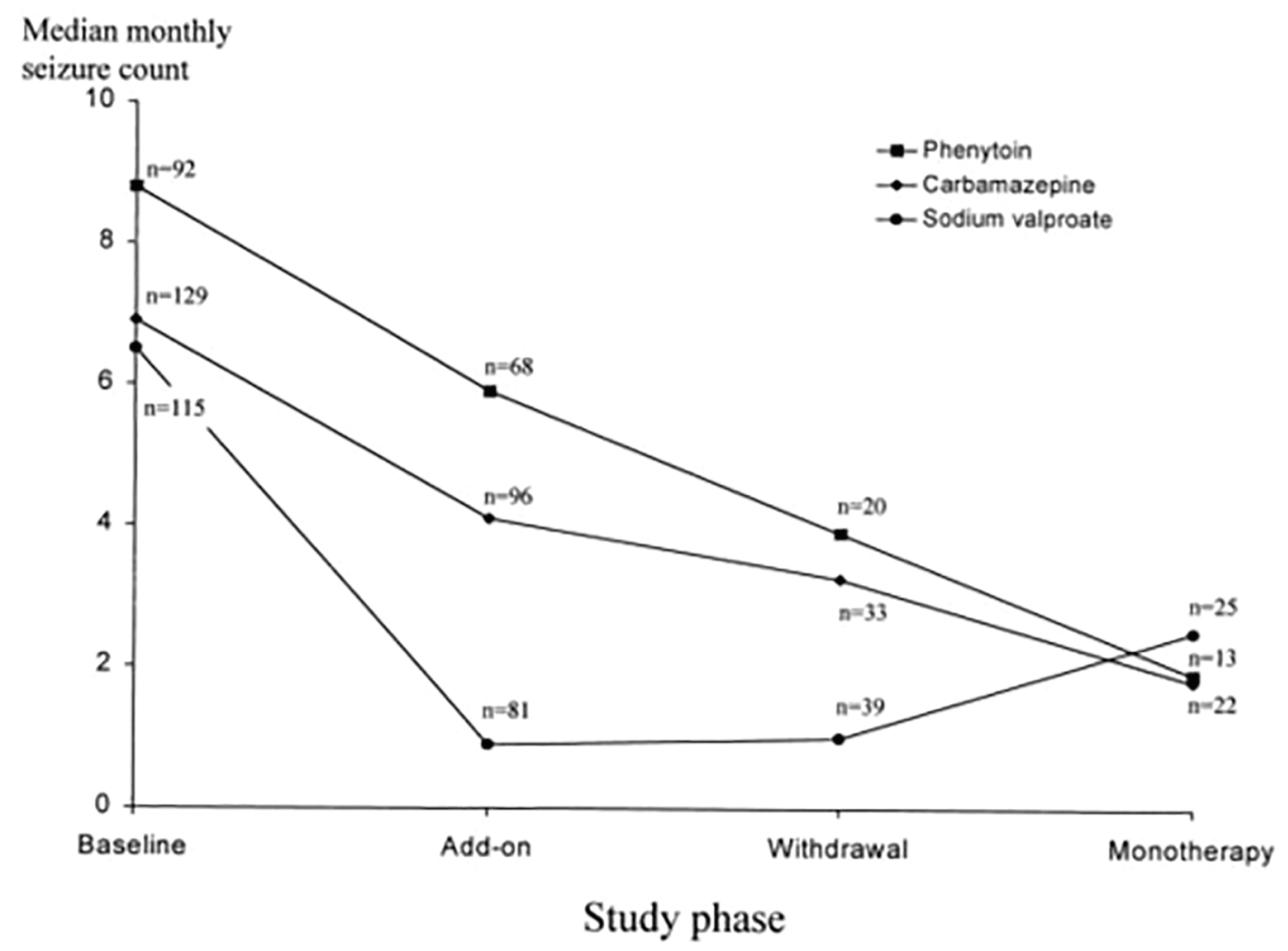

Figure 2. Lamotrigine substitution for each of the above AEDs [55].

second design suggests the add-on of the investigated AED to various other single AEDs [29]. Regarding combinations of AEDs, the following question arises: Do patients respond better to the combination of two drugs given after each failed as monotherapy? Some studies have addressed this question, investigating combinations of CBZ with valproate [49], PHT with CBZ [50], lamotrigine with valproate [51], valproate with ethosuximide [52] and vigabatrin with CBZ [53]. These studies indicate that patients who have failed to respond to these drugs as a monotherapy may respond to combinations. The following questions need to be addressed: What is the aim of combination therapy? Is polytherapy more efficient than monotherapy for newly diagnosed epilepsy if controlled for total drug load? In most clinical trials, two drugs are combined at a standard dose to produce additive efficacy. However, in some trials, the aim of combination therapy may be a reduction of side effects like toxicity rather than improved efficacy. The second question has been addressed by Deckers in a study on patients with untreated partial or generalized tonic-clonic seizures, who received treatment either as a monotherapy (400 mg of CBZ per day) or a combination of valproate and CBZ with an equivalent drug load (200 mg and $300 \mathrm{mg}$ per day respectively). After a year, no significant differences were observed between the two treatments in terms of seizure frequency or side effects $[13,54]$.

From clinical studies on humans, is it easy and straightforward to identify additive and supra-additive efficacy? Definitive data supporting the existence of additive or supra-additive pharmacodynamic interactions are difficult to find. Patsalos et al indicate that there is a possibility that "some of these therapeutic enhancements result from pharmacokinetic interactions taking place in the central brain compartment, rather than as a result of pharmacodynamic interactions" [15].

Are there specific add-on therapies or drug combinations that are more successful than others? There are some studies on the combination of lamotrigine with valproate, which provide evidence that there is a synergistic effect. The first study was carried out by Brodie and Yuen; this remarkable study was not specifically designed to investigate the efficacy of combination therapy on 347 patients with refractory epilepsy. Lamotrigine was added to valproate, CBZ, and PHT monotherapy. If patients had more than $50 \%$ reduction in seizures, then the first AED was withdrawn. When the primary drug (PHT or CBZ) was withdrawn, seizure frequency decreased slightly. This reduction in seizure frequency was possibly due to removal of an enzyme-inducing drug and a resulting increase of lamotrigine serum level. In contrast, there was an increase in seizure frequency when the valproate was withdrawn, despite an increase of lamotrigine serum levels. This effect indicates that there may be some clinical benefit (may be an additive or even a supra-additive effect) of these two drugs [55] (Fig. 2).

Another study by Kanner and Frey has supported the previous evidence; this study relates to VPA, LMG, and their combinations as add-on, controlled for pharmacokinetic interactions. In this study, patients with refractory epilepsy were treated with at least three AEDs. All patients were refractory to lamotrigine monotherapy. The average seizure-free duration 
was 2.1 months on monotherapy and 6.2 months on co-medication [56].

\section{Add-on AEDs}

Most studies of additive effects of AEDs are randomized placebo-controlled add-on trials of novel AEDs. These studies indicate that there is no difference between add-on therapies in partial epilepsy; however, levetiracetam has been proved a favorable agent with the best responder rate (efficacy measure) and withdrawal rate (mainly tolerability measure) compared to several novel AEDs [57]. It can significantly decrease focal seizure frequency in both adults and children; however, the results cannot be used to confirm efficacy as monotherapy, the longer-term effects of levetiracetam, or its effects on generalized seizures [58]. In nine randomized controlled trials, topiramate, rufinamide, felbamate, and lamotrigine were shown to be helpful as add-on medications in Lennox-Gaustaut syndrome. Despite the fact that no study to date has reported any one drug to be highly efficacious in treatment of drop seizures, clobazam may be helpful for this syndrome [59]. Topiramate was found effective as an add-on therapy in adults with refractory partial epilepsy with or without secondary generalization. However, the trials reviewed were of relatively short duration and provided no evidence for the long-term efficacy of topiramate. Results cannot be extrapolated to treatments of other epilepsy types or monotherapy $[60,61]$. Oxcarbazepine, gabapentin, zonisamide, and eslicarbazepine acetate have been reported to be effective as add-on therapy for refractory partial seizure. However, the short duration of studies makes it difficult to extrapolate to long-term treatment [62-65]. Status epilepticus is considered a common neurological emergency with a high mortality rate of $20 \%$. Therefore it needs urgent intervention by intravenous $\mathrm{PB}, \mathrm{PHT} /$ fosphenytoin, valproate or levetiracetam. Currently, these are the standard treatments after failure of benzodiazepines. Several reports have recently been published on the use of lacosamide for the treatment of status epilepticus [66]. Lacosamide has been administrated intravenously since 2009 as a new drug for the treatment of status epilepticus $[67,68]$. Long-term retention studies in clinical practice may present a proper idea about the success of combination drugs. Such studies provide a significant measure of the effectiveness (efficacy and tolerability) and adverse effects of a specific drug as add-on therapy. In studies from the same center of retention rates of add-on therapy at 3 years, levetiracetam was best (56\%), in this setting, suggesting that, levetiracetam is either more efficacious or better tolerated, or both. A possible explanation for levetiracetam's unique profile in terms of effectiveness may be its distinct putative mechanism of action, followed by topiramate (30\%). More patients appear to continue with topiramate compared with lamotrigine or gabapentin, possibly because of better perceived efficacy of topiramate, despite its having the highest incidence of adverse effects. This is followed by lamotrigine (29\%) and gabapentin $(<10 \%)[13,69,70]$. These studies have not revealed any particularly efficacious combinations. However, another retention study reported that the combination of valproate and lamotrig- ine may be more successful than lamotrigine combined with other AEDs [71].

\section{Adverse effects of AED combinations}

Several studies on specific AED combinations have showed side effects. In the study by Kanner and Fery, through the combination of valproate and lamotrigine is synergistic for efficacy, it may also be synergistic for side effects, namely tremors [56]. Notably, in one paper, three patients experienced an onset of chorea upon being treated with a combination of lamotrigine and PHT. Interestingly, the chorea was resolved in all patients with the tapering of the dosage of one AED [72]. Another example is the studies that demonstrated that the combination of CBZ and vigabatrin led to increase in the adverse effects such as ataxia and weight gain [73]. Significantly, hepatic toxicity has been reported as a side effect in patients taking valproate in combination therapy instead of monotherapy [74]. Significantly, valproate can be potentiated in terms of adverse effects, such as valproate encephalopathy, by the addition of topiramate [75].

\section{Conclusion}

Although some combinations of AEDs are very effective, the results of experimental studies on animals have to be transferred with caution to the clinical arena. Nevertheless, in many cases, there is a good correlation between clinical and experimental data. Experimental data on interactions between AEDs may provide valuable clues about clinical effectiveness or adverse therapeutic effects of drug combinations. What specific combinations of AEDs are likely to be the most effective? Although promising results from the experimental point of view support the combination of topiramate separately with lamotrigine, gabapentin, and felbamate, the best evidence supports the use of valproate and lamotrigine; this combination yields encouraging results against maximal electroshock in mice. Both AEDs induce supra-additive (synergistic) anticonvulsant effect in the dose ratio 1:1 whereas the interaction in the chimney test is infra-additive (antagonist), which seems to be very positive from an experimental point of view. Although the effectiveness of this combination is supported by human data, there may be increased side effects. The novel drugs are effective as add-on therapy. At present, in clinical practice, there is some evidence that supports levetiracetam and topiramate as the most effective add-on therapies in partial and some generalized epilepsies.

\section{Abbreviations}

$\operatorname{AED}(\mathrm{s})$ : antiepileptic drug(s); LMG: lamotrigine; OXC: oxcarbazepine; LEV: levetiracetam; TPM: topiramate; GBP: gabapentin; TGB: tiagabine; VGB: vigabatrin; FPM: felbamate; ZNS: zonisamide; PGB: pregabalin; VPA: sodium valproate; ESM: ethosuximide; STP: stiripentol; CBZ: carbamaz- 
epine; CLZ: clobazam; PB: phenobarbital; PHT: phenytoin

\section{References}

1. Mattson RH. Combination therapy with antiepileptic drugs: potential advantages and problems. Antiepileptic Drugs Combination Therapy and Interactions: Cambridge University Press; 2005. p. 16-25.

2. Davidson DT, Jr., Berman BA. Phelantin for the treatment of epilepsy. J Am Med Assoc. 1956;160(9):766-768.

3. Reynolds EH, Shorvon SD, Andersen EB. [Monotherapy or polytherapy in the treatment of epilepsy]. Ugeskr Laeger. 1982;144(51):3815-3818.

4. Schmidt D. Reduction of two-drug therapy in intractable epilepsy. Epilepsia. 1983;24(3):368-376.

5. Richens A, Davidson DL, Cartlidge NE, Easter DJ. A multicentre comparative trial of sodium valproate and carbamazepine in adult onset epilepsy. Adult EPITEG Collaborative Group. J Neurol Neurosurg Psychiatry. 1994;57(6):682-687.

6. Mattson RH, Cramer JA, Collins JF. A comparison of valproate with carbamazepine for the treatment of complex partial seizures and secondarily generalized tonic-clonic seizures in adults. The Department of Veterans Affairs Epilepsy Cooperative Study No. 264 Group. N Engl J Med. 1992;327(11):765-771.

7. Mattson RH, Cramer JA, Collins JF, Smith DB, Delgado-Escueta AV, Browne TR, Williamson PD, et al. Comparison of carbamazepine, phenobarbital, phenytoin, and primidone in partial and secondarily generalized tonicclonic seizures. N Engl J Med. 1985;313(3):145-151.

8. Heller AJ, Chesterman P, Elwes RD, Crawford P, Chadwick D, Johnson AL, Reynolds EH. Phenobarbitone, phenytoin, carbamazepine, or sodium valproate for newly diagnosed adult epilepsy: a randomised comparative monotherapy trial. J Neurol Neurosurg Psychiatry. 1995;58(1):44-50.

9. St Louis EK. Monotherapy to polytherapy: antiepileptic drug conversions through the spectrum of epilepsy care. Curr Neuropharmacol. 2009;7(2):75-76.

10. Kwan P, Brodie MJ. Epilepsy after the first drug fails: substitution or add-on? Seizure. 2000;9(7):464-468.

11. Considerations on designing clinical trials to evaluate the place of new antiepileptic drugs in the treatment of newly diagnosed and chronic patients with epilepsy. Epilepsia. 1998;39(7):799-803.

12. Bourgeois BF. Combination of valproate and ethosuximide: antiepileptic and neurotoxic interaction. J Pharmacol Exp Ther. 1988;247(3):1128-1132.

13. Walker MC. Rational Polypharmacy: Which Combinations are Most Likely to Lead to Seizure Freedom? In: Trimble MR, editor. Seizure Freedom: Clinical, Research and Quality of Life Perspectives. 2006. p. 55-73.

14. Leppik IE. Monotherapy and polypharmacy. Neurology. 2000;55(11 Suppl 3):S25-29.

15. Patsalos PN, Froscher W, Pisani F, van Rijn CM. The importance of drug interactions in epilepsy therapy. Epilepsia. 2002;43(4):365-385.
16. Loscher W, Schmidt D. Which animal models should be used in the search for new antiepileptic drugs? A proposal based on experimental and clinical considerations. Epilepsy Res. 1988;2(3):145-181.

17. Lason W, Dudra-Jastrzebska M, Rejdak K, Czuczwar SJ. Basic mechanisms of antiepileptic drugs and their pharmacokinetic/pharmacodynamic interactions: an update. Pharmacol Rep. 2011;63(2):271-292.

18. Hewlett PS. Measurement of the potencies of drug mixtures. Biometrics. 1969;25(3):477-487.

19. Gessner PK. Isobolographic analysis of interactions: an update on applications and utility. Toxicology. 1995;105(2-3):161-179.

20. Bourgeois BFD. Methods for assessing pharmacodynamic interactions. Antiepileptic Drugs Combination Therapy and Interactions: Cambridge University Press. 2005. p. 193-207.

21. Tallarida RJ. Statistical analysis of drug combinations for synergism. Pain. 1992;49(1):93-97.

22. Czuczwar SJ. Experimental studies of pharmacodynamic interactions. Antiepileptic Drugs Combination Therapy and Interactions: Cambridge University Press; 2005. p. 208-227.

23. White HS, Smith MD, Wilcox KS. Mechanisms of action of antiepileptic drugs. Int Rev Neurobiol. 2007;81:85110.

24. Rogawski MA, Porter RJ. Antiepileptic drugs: pharmacological mechanisms and clinical efficacy with consideration of promising developmental stage compounds. Pharmacol Rev. 1990;42(3):223-286.

25. Schmidt D. Two antiepileptic drugs for intractable epilepsy with complex-partial seizures. J Neurol Neurosurg Psychiatry. 1982;45(12):1119-1124.

26. Goldsmith P, de Bittencourt PR. Rationalized polytherapy for epilepsy. Acta Neurol Scand Suppl. 1995;162:35-39.

27. Pollard JR, and Jacqueline French. "Clinical studies of pharmacodynamic interactions", Antiepileptic Drugs. 1st ed. Cambridge: Cambridge University Press, 2005. 228240. 2005.

28. Reife RA. Assessing pharmacokinetic and pharmacodynamic interactions in clinical trials of antiepileptic drugs. Adv Neurol. 1998;76:95-103.

29. Deckers CL, Czuczwar SJ, Hekster YA, Keyser A, Kubova H, Meinardi H, Patsalos PN, et al. Selection of antiepileptic drug polytherapy based on mechanisms of action: the evidence reviewed. Epilepsia. 2000;41(11):13641374.

30. Deckers CL, Hekster YA, Keyser A, Meinardi H, Renier WO. Drug load in clinical trials: a neglected factor. Clin Pharmacol Ther. 1997;62(6):592-595.

31. Bourgeois BFD. Pharmacodynamic principles and mechanisms of drug interactions. Antiepileptic Drugs Combination Therapy and Interactions: Cambridge University Press. 2005. p. 181-192.

32. Bourgeois BF. Antiepileptic drug combinations and experimental background: the case of phenobarbital and phenytoin. Naunyn Schmiedebergs Arch Pharmacol. 1986;333(4):406-411.

33. Masuda Y, Utsui Y, Shiraishi Y, Karasawa T, Yoshida K, 
Shimizu M. Evidence for a synergistic interaction between phenytoin and phenobarbital in experimental animals. J Pharmacol Exp Ther. 1981;217(3):805-811.

34. Weaver LC, Swinyard EA, Woodbury LA, Goodman LS. Studies on anticonvulsant drug combinations: phenobarbital and diphenylhydantoin. J Pharmacol Exp Ther. 1955;113(3):359-370.

35. Bourgeois BF. Anticonvulsant potency and neurotoxicity of valproate alone and in combination with carbamazepine or phenobarbital. Clin Neuropharmacol. 1988;11(4):348359.

36. Chez MG, Bourgeois BF, Pippenger CE, Knowles WD. Pharmacodynamic interactions between phenytoin and valproate: individual and combined antiepileptic and neurotoxic actions in mice. Clin Neuropharmacol. 1994;17(1):32-37.

37. Bourgeois BF, Wad N. Combined administration of carbamazepine and phenobarbital: effect on anticonvulsant activity and neurotoxicity. Epilepsia. 1988;29(4):482487.

38. Shank RP, Gardocki JF, Vaught JL, Davis CB, Schupsky JJ, Raffa RB, Dodgson SJ, et al. Topiramate: preclinical evaluation of structurally novel anticonvulsant. Epilepsia. 1994;35(2):450-460.

39. Luszczki JJ, Borowicz KK, Swiader M, Czuczwar SJ. Interactions between oxcarbazepine and conventional antiepileptic drugs in the maximal electroshock test in mice: an isobolographic analysis. Epilepsia. 2003;44(4):489499.

40. Borowicz KK, Luszczki JJ, Sobieszek G, Ratnaraj N, Patsalos PN, Czuczwar SJ. Interactions between zonisamide and conventional antiepileptic drugs in the mouse maximal electroshock test model. Eur Neuropsychopharmacol. 2007;17(4):265-272.

41. Luszczki JJ, Czuczwar M, Kis J, Krysa J, Pasztelan I, Swiader M, Czuczwar SJ. Interactions of lamotrigine with topiramate and first-generation antiepileptic drugs in the maximal electroshock test in mice: an isobolographic analysis. Epilepsia. 2003;44(8):1003-1013.

42. Dudra-Jastrzebska M, Andres-Mach MM, Ratnaraj N, Patsalos PN, Czuczwar SJ, Luszczki JJ. Isobolographic characterization of the anticonvulsant interaction profiles of levetiracetam in combination with clonazepam, ethosuximide, phenobarbital and valproate in the mouse pentylenetetrazole-induced seizure model. Seizure. 2009; 18(9):607-614.

43. Luszczki JJ, Czuczwar SJ. Biphasic characteristic of interactions between stiripentol and carbamazepine in the mouse maximal electroshock-induced seizure model: a three-dimensional isobolographic analysis. Naunyn Schmiedebergs Arch Pharmacol. 2006;374(1):51-64.

44. Luszczki J, Swiader M, Czuczwar M, Kis J, Czuczwar SJ. Interactions of tiagabine with some antiepileptics in the maximal electroshock in mice. Pharmacol Biochem Behav. 2003;75(2):319-327.

45. Luszczki JJ, Krzyzanowski M, Swiader MJ. Interaction of tiagabine with valproate in the mouse pentylenetetrazole-induced seizure model: an isobolographic analysis for non-parallel dose-response relationship curves. Adv
Med Sci. 2009;54(1):75-81.

46. Luszczki JJ. Isobolographic analysis of interaction between drugs with nonparallel dose-response relationship curves: a practical application. Naunyn Schmiedebergs Arch Pharmacol. 2007;375(2):105-114.

47. Luszczki JJ, Czuczwar SJ. Preclinical profile of combinations of some second-generation antiepileptic drugs: an isobolographic analysis. Epilepsia. 2004;45(8):895-907.

48. Borowicz KK, Swiader M, Luszczki J, Czuczwar SJ. Effect of gabapentin on the anticonvulsant activity of antiepileptic drugs against electroconvulsions in mice: an isobolographic analysis. Epilepsia. 2002;43(9):956-963.

49. Walker JE, Koon P. Carbamazepine versus valproate versus combined therapy for refractory partial seizures with secondary generalization. Epilepsia. 1988;29:693.

50. Hakkarainen H. Carbamazepine vs. diphenylhydrantoin vs. their combination in adult epilepsy. Neurology. 1980;30:354.

51. Moeller JJ, Rahey SR, Sadler RM. Lamotrigine-valproic acid combination therapy for medically refractory epilepsy. Epilepsia. 2009;50(3):475-479.

52. Rowan AJ, Meijer JW, de Beer-Pawlikowski N, van der Geest P, Meinardi H. Valproate-ethosuximide combination therapy for refractory absence seizures. Arch Neurol. 1983;40(13):797-802.

53. Tanganelli P, Regesta G. Vigabatrin vs. carbamazepine monotherapy in newly diagnosed focal epilepsy: a randomized response conditional cross-over study. Epilepsy Res. 1996;25(3):257-262.

54. Deckers CL, Hekster YA, Keyser A, van Lier HJ, Meinardi H, Renier WO. Monotherapy versus polytherapy for epilepsy: a multicenter double-blind randomized study. Epilepsia. 2001;42(11):1387-1394.

55. Brodie MJ, Yuen AW. Lamotrigine substitution study: evidence for synergism with sodium valproate? 105 Study Group. Epilepsy Res. 1997;26(3):423-432.

56. Kanner AM, Frey M. Adding valproate to lamotrigine: a study of their pharmacokinetic interaction. Neurology. 2000;55(4):588-591.

57. Otoul C, Arrigo C, van Rijckevorsel K, French JA. Metaanalysis and indirect comparisons of levetiracetam with other second-generation antiepileptic drugs in partial epilepsy. Clin Neuropharmacol. 2005;28(2):72-78.

58. Mbizvo GK, Dixon P, Hutton JL, Marson AG. Levetiracetam add-on for drug-resistant focal epilepsy: an updated Cochrane Review. Cochrane Database Syst Rev. 2012;9:CD001901.

59. Hancock EC, Cross JH. Treatment of Lennox-Gastaut syndrome. Cochrane Database Syst Rev. 2013;2:CD003277.

60. Reife R, Pledger G, Wu SC. Topiramate as add-on therapy: pooled analysis of randomized controlled trials in adults. Epilepsia. 2000;41(Suppl 1):S66-71.

61. Jette N, Hemming K, Hutton JL, Marson AG. Topiramate add-on for drug-resistant partial epilepsy. Cochrane Database Syst Rev. 2008;(3):CD001417.

62. Castillo S, Schmidt DB, White S. Oxcarbazepine add-on for drug-resistant partial epilepsy. Cochrane Database Syst Rev. 2000;(3):CD002028.

63. Chadwick DW, Marson AG. Zonisamide add-on for drug- 
resistant partial epilepsy. Cochrane Database Syst Rev. 2005;(4):CD001416.

64. Marson AG, Kadir ZA, Hutton JL, Chadwick DW. Gabapentin add-on for drug-resistant partial epilepsy. Cochrane Database Syst Rev. 2000;(3):CD001415.

65. Chang XC, Yuan H, Wang Y, Xu HQ, Zheng RY. Eslicarbazepine acetate add-on for drug-resistant partial epilepsy. Cochrane Database Syst Rev. 2011;(12):CD008907.

66. Fernandez EM, Franck AJ. Lacosamide for the treatment of refractory status epilepticus. Ann Pharmacother. 2011;45(11):1445-1449.

67. Shorvon S. The treatment of status epilepticus. Curr Opin Neurol. 2011;24(2):165-170.

68. Hofler J, Trinka E. Lacosamide as a new treatment option in status epilepticus. Epilepsia. 2013;54(3):393-404.

69. Lhatoo SD, Wong IC, Polizzi G, Sander JW. Long-term retention rates of lamotrigine, gabapentin, and topiramate in chronic epilepsy. Epilepsia. 2000;41(12):1592-1596.

70. Depondt C, Yuen AW, Bell GS, Mitchell T, Koepp MJ,
Duncan JS, Sander JW. The long term retention of levetiracetam in a large cohort of patients with epilepsy. J Neurol Neurosurg Psychiatry. 2006;77(1):101-103.

71. Knoester PD, Keyser A, Renier WO, Egberts AC, Hekster YA, Deckers CL. Effectiveness of lamotrigine in clinical practice: results of a retrospective population-based study. Epilepsy Res. 2005;65(1-2):93-100.

72. Zaatreh M, Tennison M, D'Cruz O, Beach RL. Anticonvulsants-induced chorea: a role for pharmacodynamic drug interaction? Seizure. 2001;10(8):596-599.

73. Murri L, Iudice A. Vigabatrin as first add-on treatment in carbamazepine-resistant epilepsy patients. Acta Neurol Scand Suppl. 1995;162:40-42.

74. Dreifuss FE, Santilli N, Langer DH, Sweeney KP, Moline KA, Menander KB. Valproic acid hepatic fatalities: a retrospective review. Neurology. 1987;37(3):379-385.

75. Hamer HM, Knake S, Schomburg U, Rosenow F. Valproate-induced hyperammonemic encephalopathy in the presence of topiramate. Neurology. 2000;54(1):230-232. 